\title{
New Precision Tests of the Einstein Equivalence Principle from SN1987A
}

\author{
Michael J. Longo \\ University of Michigan, Ann Arbor, Michigan 48109 \\ (Received 14 September 1987)
}

\begin{abstract}
The serendipitous observation of neutrinos and light from the recent supernova in the Large Magellanic Cloud has provided a wealth of new information about stellar collapse and about neutrinos. It is pointed out that, in addition, the nearly simultaneous arrival of the photons and neutrinos after a journey of some $160000 \mathrm{yr}$ provides a new, stringent test of the Einstein equivalence principle on intergalactic distance scales. The relativistic time delays of the photons and neutrinos from SN1987A caused by the gravitational field of our Galaxy are compared, and it is shown that they are equal, to an accuracy of approximately $0.2 \%$ of the predicted delay.

PACS numbers: $04.80 .+\mathrm{z}, 97.60 . \mathrm{Bw}$
\end{abstract}

The nearly simultaneous detection of light ${ }^{1}$ and neutri$\operatorname{nos}^{2}$ from the supernova SN1987A has provided us with an important verification of theories of stellar collapse. ${ }^{3}$ The arrival of neutrinos with a wide range of energies in a period of only a few seconds, after having traveled some $50 \mathrm{kpc}$, also leads to a useful upper limit on the electron-neutrino mass. ${ }^{4}$

In addition to these important results, SN1987A also provides us with a unique probe of space-time on intergalactic scales. The fact that the transit times of photons and neutrinos from SN1987A to the Earth are the same within several hours in $\simeq 1.6 \times 10^{5} \mathrm{yr}$ yields a new high-precision test of special relativity and of the Einstein equivalence principle (EEP) which is a fundamental postulate of general relativity and other metric theories of gravity. The test of special relativity, a comparison of the velocity of neutrinos to that of light to an accuracy of $2 \times 10^{-9}$, was discussed in an earlier note. ${ }^{5}$ Here I discuss the implications for the EEP and general relativity.

There are few precise tests of the EEP and general relativity. Among the most precise are the measurement of the gravitational deflection of light or radio waves near the Sun and the measurement of the time delay of radio waves due to the Sun's gravity. ${ }^{6,7}$ None of these tests involve neutrinos, and all are confined to the solar system (except an indirect argument which links the rate at which a compact binary pulsar slows down due to the emission of gravitational waves).

As is shown below, the gravitational field of our galaxy causes a significant time delay, $\approx 5$ months, in the transit time of photons from SN1987A. (This is the delay relative to the transit time expected if the gravitation of the galaxy could be "turned off.") The fact that the arrival time of the neutrinos from SN1987A was the same as that for the first optical photons from the supernova to within several hours allows an accurate comparison of the general-relativistic time delay of the photons and neutrinos. The arrival time of the neutrinos is known to be within a few seconds of 7:35:40 UT on 23 February. ${ }^{2}$
The arrival time of the first light from the supernova is less well known. The last confirmed evidence of no optical brightening ${ }^{8}$ was by Shelton ${ }^{1 c}$ at approximately 2:20 UT. The earliest observations of optical brightening were by Garradd $^{1 \mathrm{c}}$ and by McNaught ${ }^{\text {lc }}$ at 10:38 UT. It is also expected that the neutrinos are emitted in the first few seconds of the collapse, while the outburst of visible light begins a few hours later when the cooler envelope is blown away.

From these observations we can conclude that the first light arrived some time between about $5 \mathrm{~h}$ before the neutrinos ${ }^{8}$ and $3 \mathrm{~h}$ after. However, the time difference between the emission of the neutrinos and the optical brightening at the source is somewhat controversial. ${ }^{9}$ It is possible that the neutrinos were emitted, say, $20 \mathrm{~h}$ earlier than the outburst of visible light, but they were delayed in transit by a larger relativistic time delay and so arrived here about the same time as the first photons. To be reasonably conservative let us therefore assume that the uncertainty in the relative transit time of photons and neutrinos from SN1987A is approximately $\pm 6 \mathrm{~h}$.

If the EEP is correct, both the photons and neutrinos from SN1987A will incur exactly the same time delay due to gravitational fields in the intervening space. The mass of the Large Magellanic Cloud is estimated to be $<3 \%$ of the mass of the galaxy, ${ }^{10}$ and so the effect of its gravitation can be neglected for my purposes. The photons and neutrinos from SN1987A spend most of the time outside our galaxy and so it is reasonable to approx-

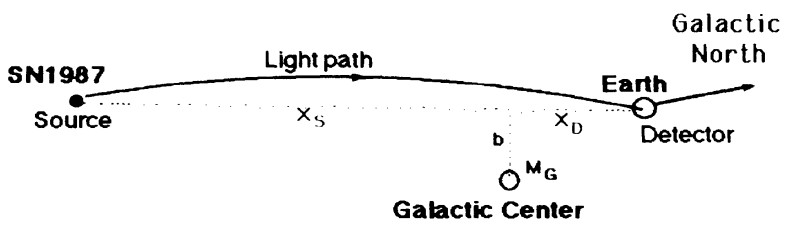

FIG. 1. Geometry and notation used in the time-delay calculation. $\left(X_{D}, b\right.$, and the curvature of the light path are exaggerated for clarity.) 
imate our galaxy as a point mass at its center in the calculation of the expected time delay. The time delay can then be calculated in exactly the same fashion as that for radar waves passing near the sun. ${ }^{6}$ The geometry and notation are shown in Fig. 1. The deviation of the path from a straight line can be neglected in the time-delay calculation.

In units where the gravitational constant $G$ and the velocity of light are unity, the time delay for light is ${ }^{6}$

$$
\delta t_{\gamma}=\left(1+\gamma_{\gamma}\right) M_{G} \ln \left\{\left[X_{S}+\left(X_{S}^{2}+b^{2}\right)^{1 / 2}\right]\left[X_{D}+\left(X_{D}^{2}+b^{2}\right)^{1 / 2}\right] / b^{2}\right\} .
$$

Here $M_{G}$ is the mass of the galaxy. In the standard "parametrized post-Newtonian" formalism, ${ }^{6,7} \gamma$ represents how much space curvature is produced by unit rest mass. In general relativity, $\gamma=1$. The subscript $\gamma$ is used to emphasize that this parameter need not be the same for photons and neutrinos-their time delays may differ if the EEP fails. Thus the ratio $\left(\delta t_{\gamma}-\delta t_{v}\right) / \delta t_{\gamma}$ $\approx \frac{1}{2}\left(\gamma_{\gamma}-\gamma_{v}\right)$ can be used as a measure of a possible violation of the EEP.

It is important to note that all metric theories of gravity incorporate the EEP and so they all predict that photons and neutrinos must follow identical trajectories and undergo the same time delay, though their predictions for this delay may differ. In other words, all metric theories predict $\gamma_{\gamma}=\gamma_{\nu} \equiv \gamma$ but $\gamma$ need not be 1. Just as in the case of the gravitational deflection of light, ${ }^{7}$ half of the delay in Eq. (1) is caused by the warping of space by the gravitational field of the galaxy (the $\gamma$ term); the other half can be thought of as a result of a gravitational red shift. ${ }^{11}$

The total mass of our galaxy is not well known. We use the galactic model calculation of Bahcall, Schmidt, and Soneira. ${ }^{12}$ They estimate the total mass of the galaxy inside $60 \mathrm{kpc}$ to be about $6 \times 10^{11} M_{\odot}$, mostly in the halo. In convenient units, the mass of the sun $M_{\odot}$ is $4.9 \mu \mathrm{s}$. The impact parameter of the rays $b$ (Fig. 1) is on the order of the distance of the sun from the center of the galaxy, approximately $12 \mathrm{kpc}$. Since $X_{D} \ll b$, and $X_{S} \gg b$, the logarithmic factor in Eq. (1) is $\approx \ln \left(2 X_{S} /\right.$ $b$ ), where $X_{S}$ is the distance to the Large Magellanic Cloud, approximately $50 \mathrm{kpc}{ }^{10}$ Thus the expected time delay for light is

$$
\delta t_{\gamma} \approx 6 \times 10^{11} M_{\odot}\left(1+\gamma_{\gamma}\right) \ln (2 \times 50 / 12) \approx 6 \times 10^{6}\left(1+\gamma_{\gamma}\right) \mathrm{s},
$$

or about 4.8 months if $\gamma_{\gamma}=1$. Since the arrival time of the photons and neutrinos is known to be equal to within about $6 \mathrm{~h}$,

$$
\left(\delta t_{\gamma}-\delta t_{v}\right) / \delta t_{\gamma}=\frac{1}{2}\left(\gamma_{\gamma}-\gamma_{v}\right) \lesssim 6 \mathrm{~h} / 4.8 \text { months }=1.7 \times 10^{-3},
$$

and

$$
\gamma_{\gamma}-\gamma_{v} \lesssim 3.4 \times 10^{-3} \text {. }
$$

Thus we can conclude that neutrinos and photons "see" the same gravitationally induced time delay to about $0.2 \%$. As discussed above, half of the time delay comes from the curvature of space due to the gravitational field of the galaxy and half from a gravitational red shift. Thus both aspects of the theory are tested.

It is also possible that neutrinos (or photons) of different energies can respond differently to the space curvature due to our galaxy. The very small dispersion in arrival times observed for neutrinos of widely different energies can therefore be used to set a limit on any energy dependence of $\gamma_{v}$. The dispersion in arrival times ${ }^{2}$ for neutrinos ranging in energy from about 7.5 to $40 \mathrm{MeV}$ was observed to be less than approximately $10 \mathrm{~s}$. Thus

$$
\left[\delta t_{v}(40 \mathrm{MeV})-\delta t_{v}(7.5 \mathrm{MeV})\right] / \delta t_{\gamma} \leqslant 10 \mathrm{~s} / 4.8 \text { months }=8 \times 10^{-7},
$$

so that

$$
\left[\gamma_{v}(40 \mathrm{MeV})-\gamma_{v}(7.5 \mathrm{MeV})\right] \lesssim 1.6 \times 10^{-6} .
$$

(Of course, it is possible that an energy dependence of the relativistic time delay could be accidentally compensated by a time delay due to a small rest mass of the neutrino.)

On the basis of the SN1987 neutrino and optical data we can therefore conclude that, as predicted by the EEP, the gravitationally induced time delays of neutrinos and photons are equal and there is no dependence of this time delay on neutrino energy to a very high accuracy. This fortuitous supernova provides us with a new test showing that neutrinos and photons of widely different energies all follow the same geodesic paths in the curved spacetime in and near our galaxy.

Useful conversations with P. V. R. Murthy are gratefully acknowledged. This work was supported in part by a grant from the National Science Foundation.

\footnotetext{
1aI. Shelton, International Astronomical Union (IAU) Circular No. 4316 (1987).

${ }^{\text {lb }}$. H. McNaught, IAU Circular No. 4316 (1987); G. Garradd, IAU Circular No. 4316 (1987).
} 
${ }^{1}$ I. Shelton, IAU Circular No. 4330 (1987).

${ }^{2}$ K. Hirata et al., Phys. Rev. Lett. 58, 1490 (1987); R. M. Bionta et al., Phys. Rev. Lett. 58, 1494 (1987).

${ }^{3}$ See, for example, J. N. Bahcall, A. Dar, and T. Piran, Nature (London) 326, 135 (1987); D. N. Schramm, R. Mayle, and J. R. Wilson, Nuovo Cimento 9C, 493 (1986).

${ }^{4}$ See, for example, W. Arnett and J. Rosner, Phys. Rev. Lett. 58, 1906 (1987).

${ }^{5}$ M. J. Longo, Phys. Rev. D 36, 3276 (1987).

${ }^{6}$ C. W. Misner, K. S. Thorne, and J. A. Wheeler, Gravitation (Freeman, San Francisco, 1973).

${ }^{7}$ Clifford M. Will, Phys. Rep. 113, 345 (1984), and Theory and Experiment in Gravitational Physics (Cambridge Univ. Press, Cambrige, 1981).

${ }^{8}$ There is a possible observation of no optical brightening of SN1987A at 09:22 UT by A. Jones [IAU Circular No. 4340 (1987)]. This would restrict the arrival time of the first light to a much narrower window, 09:22 to $10: 38$. This in turn would give some improvement on the limit (2). However, to be conservative I use the less restrictive limit based on Ref. 1c.

${ }^{9}$ A de Rújula [Phys. Lett. B 193, 514 (1987)] discusses the possibility that two neutrino bursts spaced by several hours actually occurred, and he emphasizes the uncertainty in the theoretical calculations of the neutrino burst(s) and the optical flare. In his own "two-bang" scenario he concludes that the light curve begins to rise within tens of minutes after the first neutrino burst.

${ }^{10}$ A. D. Thackeray, in The Large Magellanic Clouds, edited by A. Muller (Springer-Verlag, New York, 1971).

${ }^{11}$ Gedankenexperiments which link the gravitational time delay and the deflection of light to the equivalence principle are described in Clifford M. Will, Was Einstein Right?: Putting General Relativity to the Test (Basic Books, Inc. New York, 1986).

12J. Bahcall, M. Schmidt, and R. M. Soneira, Astrophys. J. 265, 730 (1983). 